\title{
Chapter 2 of Network Performance and Quality of Service: Determination of Key Performance Indicator (KPI)
}

Paper DOI:10.17605/OSF.IO/6GTND

Citation: Haryadi, S. (2018, January 26). Chapter 2 of Network Performance and Quality of Service: Determination of Key Performance Indicator (KPI). Retrieved from osf.io/preprints/inarxiv/6gtnd

\section{The contents of Telecommunication Service Performance Indicators [20]}

The performance indicators of telecommunications services consist of two inseparable things, that the performance parameters and the target value to be achieved on any parameter. Performance parameters are parameters that represent performance, there are two possible states, first in a positive statement, e.g. "availability", then the target value in this case the minimum value to be achieved, e.g. 95\%. However, it can also be a parameter of performance in the form of a negative statement, e.g. "unavailability" then in this case the target value is the maximum number that can be reached, referring to the target value availability is $5 \%$.

Basically, the performance parameters of telecommunications services are very many, refer to Chapter 1 of this book, there are many ways to categorize the performance parameters. Thus, there needs a guidance to composing a performance indicator groups are considered the most important to have become a KPI (Key Performance Indicator).

\section{Procedure of Key Performance Indicators Determination}

Technical considerations determining the QoS is also very complicated, as it include the selection of transport technology, topology, and reliability of the device. 
Providers should develop the KPI (Key Performance Indicator), where KPI should arranged on several considerations:

1) Should be fulfilled and is determined as the minimum standards for the telecommunications provider is a standard defined by the National Regulatory Body.

2) The company's performance standards should be wider (more performace parameters with better target values) than the standards set by the National Regulatory Body. For that purpose, the provider must learn and referring to the international standards and Recommendations of the ITU-T, ETSI, IEEE, ANSI and others.

3) Because the world recommendation or standardization is too many, the operator should elects the parameters which they think are the most important as the key parameter. For its task, is necessary to do the benchmarking with the standards of various world class telecom operators and National Regulatory Bodies in the world, and each performance parameter is accompanied by a target value. The key parameters must be ensured is in the agreement between the provider with the vendors.

4) Last but not least, the last consideration is the SLA (service level agreement) between providers and customers.

\section{NOTE:}

1) ITU-T is an agency under the United Nations, thus being the most fair of reference materials, all member countries, should pay attention to the recommendations of ITU-T. But, the ITU-T Recommendations are too many, thus every provider should have some staff, which specifically follows the state of the art of the recommendations issued by the ITU-T, particularly with respect to the performance of telecommunications networks and services.

2) The telecommunications regulatory body in each country has different quality, when the regulator is good, then it was able to formulate the key performance indicator of telecommunications services that should be fulfilled by every providers. The meaning of $A$ good rule is that able to be met by the provider and also 
satisfying the customers. Unfortunately, the customer satisfaction is influenced by the rates they pay, as an example: the customers will make a complain to the providers when they pay more expensive than other provider's rates, eventhough the performance is better.

3) SLA is one of the reasons that the provider should monitor the telecommunication traffic at every moment, in every place and at every service. Through SLA, the provider promises to serve customers by meeting specific performance requirements, while the SLA is not met, than the payment cannot be obtained, or may even the provider have to pay a penalty to its customers.

\section{Target Values Determination}

After selecting the KPI parameters, then we have to determines the target value of each parameter. Unfortunately, the determination of the target values are certainly needs the long time and often the target values should be change to adapts to the business and technology situation.

Here are some guidelines for determining the target values.

1) The target value of the KPI provider parameters should be higher than the target value set by the regulatory body. For example, regulators set a target value for chat success of $90 \%$ and end to-end delay is not allowed to exceed 1 minute, then the provider sets the target value, for example, are: $95 \%$ and 30 seconds.

2) The provider should evaluates the target value in about 6 months to 1 year:

a. The provider increase the target if there are many complaints from customers in its performace indicator. 


\section{Examples of the KPI (Key Performance Indicator)}

Examples of KPI parameters are shown in the following table:

Table 1. The Example of Key Performance Indicator and Its target Value

\begin{tabular}{|c|c|c|}
\hline Type of KPI & PERFORMANCE PARAMETER & TARGET VALUE \\
\hline $\begin{array}{l}\text { General Requirement of } \\
\text { the World Class } \\
\text { Operators (benchmark) }\end{array}$ & $\begin{array}{ll}\text { - } & \text { Activation time of services } \\
\text { - } & \text { Malfunctioning rate of services for } \\
\text { - } & \text { access to broadband internet } \\
\text { Repair time for services for access } \\
\text { to broadband internet } \\
\text { - } \text { Answer times to calls to customer } \\
\text { assistance services } \\
\text { - } \quad \text { Bills challenged } \\
\text { - } \quad \text { Quality of Customer Experience } \\
\text { Customer Care Services }\end{array}$ & $\begin{array}{l}\text { - } 10 \text { minutes - one hour } \\
\text { - } 1 \text { minutes - } 10 \text { minutes } \\
\text { - } 30 \text { minutes - } 3 \text { hours } \\
\text { - } \quad 1-5 \text { seconds } \\
\text { - } \quad 0.00001 \text { maximum error } \\
\text { - } 95-99 \% \\
\text { - } 95-99 \%\end{array}$ \\
\hline $\begin{array}{l}\text { Telephony Service } \\
\text { Quality }\end{array}$ & Mean Opinion Score (MOS) & $\begin{array}{l}4-4.5(\text { maximum scale }=5) \text { or } \\
3.75-4.25(\text { maximum }=4.5)\end{array}$ \\
\hline $\begin{array}{l}\text { General Requirement of } \\
\text { National Regulatory } \\
\text { Bodies (benchmark) }\end{array}$ & $\begin{array}{l}\text { - } \quad \text { Service Coverage } \\
\text { - } \quad \text { Network availability } \\
\text { - } \quad \text { Network Congestion (During } \\
\text { Busy Hour) } \\
\text { - } \quad \text { Success rate of Telephony } \\
\text { Calls } \\
\text { - } \quad \text { Set-up time for telephony calls } \\
\text { - } \quad \text { Drop call rate during busy hour } \\
\text { - } \quad \text { Complaints on coverage per } \\
\text { 1000 subscriber } \\
\text { - } \quad \text { Service availability } \\
\text { - } \quad \text { Service accessibility } \\
\text { - } \quad \text { Metering and Billing error } \\
\text { - } \quad \text { Response time to the customer } \\
\text { for assistance }\end{array}$ & $\begin{array}{ll}\cdot & 75-100 \% \\
\cdot & 95-99 \% \\
\text { - } & 0.5-2 \% \\
\text { - } & 80-98 \% \\
& \\
\cdot & 4-10 \text { seconds } \\
\text { - } & 0.5-2 \% \\
\text { - } & 0.1-1 \\
\cdot & 1 \\
\cdot & 95-99 \% \\
\cdot & 92-99 \% \\
\cdot & 0.000001 \text { (maximum) } \\
\text { - } & 2-30 \text { seconds }\end{array}$ \\
\hline
\end{tabular}


Table 2. Mobile Network Parameter Indicators

\begin{tabular}{|c|c|}
\hline Type of Service & Examples of QoS Parameter Indicator \\
\hline Network Accessibility & NER (Network Effectiveness Ratio) \\
\hline \multirow[t]{3}{*}{ Telepbony Network } & SCR (Successful Call Ratio) \\
\hline & CCR (Call Completion ratio) \\
\hline & NNGoS (End to end Grade of Service) \\
\hline \multirow{4}{*}{$\begin{array}{ll}\text { Mobile } & \text { Telephony } \\
\text { Network } & \\
\text { Accessibility } & \end{array}$} & Location Update Success Rate \\
\hline & Handover Success Rate \\
\hline & Radio Network Unavailability \\
\hline & Mean Data Rate \\
\hline \multirow[t]{3}{*}{ Telephony Service } & Telephony Setup Time [s], In the past, often referred to PDD (Post Dial Delay) \\
\hline & Telephony Speech Quality or MoS (Mean Opinion Score) \\
\hline & Telephony Cut-off Ratio \\
\hline \multirow[t]{2}{*}{ SMS } & SMS End-to-End Delivery Time \\
\hline & Completion Rate or SMS loss Percentage \\
\hline \multirow[t]{2}{*}{ Internet Accessibility } & PDP Context Activation Failure Ratio \\
\hline & Attach Failure Ratio \\
\hline \multirow[t]{5}{*}{ Internet Service } & HTTP Service Non-Accessibility \& Session Failure Ratio \\
\hline & FTP \{Download.Upload $\}$ Service Non-Accessibility \& Session Failure Ratio \\
\hline & E-Mail \{Upload|Download\} Login Non Accessibility Session Failure Ratio \\
\hline & Ping Round Trip Time \\
\hline & Streaming Service Non-Accessibility \& Reproduction Cut-off Ratio \\
\hline
\end{tabular}

\section{Details of Quality Parameters in the Various International Standards}

There are many international standards for telecommunication quality and experience, below are the summary.

Table 3. The Number of QoS parameters in Various International Institution

\begin{tabular}{|c|c|c|c|c|c|c|c|c|c|}
\hline $\begin{array}{c}\text { Service } \\
\text { Category }\end{array}$ & $\begin{array}{r}\text { Number } \\
\text { of KPI }\end{array}$ & $\begin{array}{c}\text { ETSI } \\
\text { EG } \\
202 \\
009\end{array}$ & $\begin{array}{r}\text { ETSI } \\
\text { EG } \\
202 \\
057\end{array}$ & $\begin{array}{r}\text { ETSI } \\
\text { TS } \\
102 \\
250\end{array}$ & $\begin{array}{l}\text { ITU } \\
\text { T } \\
\text { E.800 }\end{array}$ & $\begin{array}{c}\text { 3GPP } \\
\text { TS } 32 \\
410\end{array}$ & $\begin{array}{l}\text { GSMA } \\
\text { PRD } \\
\text { IR.42 }\end{array}$ & $\begin{array}{l}\text { ITU- } \\
\text { T } \\
\text { E.771 }\end{array}$ & $\begin{array}{r}\text { 3GPP } \\
52402\end{array}$ \\
\hline $\begin{array}{l}\text { Telephony } \\
\& \\
\text { Video } \\
\text { Telephony }\end{array}$ & 78 & 7 & 9 & 22 & 0 & 36 & 12 & 4 & 0 \\
\hline $\begin{array}{l}\text { SMS \& } \\
\text { MMS }\end{array}$ & 15 & 0 & 3 & 12 & 0 & 0 & 4 & 0 & 0 \\
\hline Internet & 119 & 19 & 10 & 77 & 0 & 0 & 6 & 0 & 0 \\
\hline $\begin{array}{l}\text { Customer } \\
\text { Support \& } \\
\text { Maintenance }\end{array}$ & 26 & 13 & 14 & 0 & 0 & 0 & 0 & 0 & 0 \\
\hline Total & 238 & 39 & 39 & 111 & 0 & 36 & 22 & 4 & 0 \\
\hline
\end{tabular}




\subsection{ITU-T QoS Parameters}

In the ITU-T RecommendationE.800, quality of service terms are classified

into three broad areas: service, network and management.

Table 4 Quality of Service Terms in ITU-T E.800 [5], [6]

\begin{tabular}{|c|c|c|}
\hline Service QoS Parameters & Network QoS Parameters & Management QoS Parameters \\
\hline Service-provision & Customer premises equipment & QoS resource management \\
\hline Call setup & Network/user interface & Class of service \\
\hline Call progress & Interconnection & Customer relationship management \\
\hline $\begin{array}{l}\text { Service } \\
\text { restoration/repair }\end{array}$ & Network accessibility & Benchmark \\
\hline $\begin{array}{ll}\begin{array}{l}\text { Service } \\
\text { characteristics }\end{array} & \text { quality }\end{array}$ & Connection accessibility & Compensation schemes \\
\hline Charging \& Billing & $\begin{array}{l}\text { Connection establishment error } \\
\text { probability }\end{array}$ & Service level agreement (SLA) \\
\hline Common & $\begin{array}{l}\text { Connection establishment } \\
\text { failure probability }\end{array}$ & Service quality agreement (SQA) \\
\hline Service-specific terms & $\begin{array}{l}\text { Unacceptable transmission } \\
\text { probability }\end{array}$ & Time between interruptions \\
\hline \multirow[t]{14}{*}{ Security-specific terms } & No tone probability & Interruption duration \\
\hline & Misrouting probability & Mean time between interruptions \\
\hline & Traffic ability performance & $\begin{array}{l}\text { Mean time to restoration, Mean time to } \\
\text { recovery, Mean time to repair }\end{array}$ \\
\hline & Transmission performance & Fault coverage \\
\hline & Bit transparency & Repair coverage \\
\hline & Bit error ratio (BER) & Fault \\
\hline & Error free seconds ratio & Corrective maintenance repair \\
\hline & Propagation performance & Reliability performance \\
\hline & End-to-end IP network & Failure rate acceleration factor \\
\hline & $\begin{array}{l}\text { End-to-end IP network } \\
\text { performance }\end{array}$ & Disaster recovery, Business continuity \\
\hline & $\begin{array}{l}\text { Percent IP service availability } \\
\text { (PIA) }\end{array}$ & Complaint \\
\hline & Release failure probability & Discovery service \\
\hline & $\begin{array}{l}\text { Connection clearing failure } \\
\text { probability }\end{array}$ & \\
\hline & $\begin{array}{l}\text { Connection establishment } \\
\text { success ratio (CESR) }\end{array}$ & \\
\hline
\end{tabular}


Table 5 the ITU-T Performance targets for audio, video, and data applications [5], [6]

\begin{tabular}{|c|c|c|c|c|c|c|c|}
\hline \multirow[t]{2}{*}{ Medium } & \multirow[t]{2}{*}{ Application } & \multirow{2}{*}{$\begin{array}{c}\text { Degree } \\
\text { of } \\
\text { Symmet } \\
\text { ry } \\
\end{array}$} & \multirow{2}{*}{$\begin{array}{l}\text { Typica } \\
\text { I Data } \\
\text { Rates }\end{array}$} & \multicolumn{4}{|c|}{ Key Performance Parameters \& Target Values } \\
\hline & & & & $\begin{array}{l}\text { One-way } \\
\text { delay }\end{array}$ & $\begin{array}{l}\text { Delay } \\
\text { variation }\end{array}$ & $\begin{array}{l}\text { Information } \\
\text { loss (Note 2) }\end{array}$ & Other \\
\hline Audio & $\begin{array}{l}\text { Conversational } \\
\text { voice }\end{array}$ & Two-way & $\begin{array}{l}4-64 \\
\mathrm{kbit} / \mathrm{s}\end{array}$ & $\begin{array}{l}<150 \quad \mathrm{~ms} \\
\text { preferred } \\
(\text { Note } 1) \\
<400 \mathrm{~ms} \text { limit } \\
(\text { Note } 1)\end{array}$ & $<1 \mathrm{~ms}$ & $\begin{array}{lr}<3 \% & \text { packet } \\
\text { loss } & \text { ratio } \\
(\mathrm{PLR}) & \end{array}$ & \\
\hline Audio & $\begin{array}{l}\text { Voice } \\
\text { messaging }\end{array}$ & $\begin{array}{l}\text { Primarily } \\
\text { one-way }\end{array}$ & $\begin{array}{l}4-31 \\
\mathrm{kbit} / \mathrm{s}\end{array}$ & $\begin{array}{l}<1 \quad \mathrm{~s} \text { for } \\
\text { playback } \\
<2 \mathrm{~s} \text { for record }\end{array}$ & $<1 \mathrm{~ms}$ & $<3 \%$ PLR & \\
\hline Audio & $\begin{array}{l}\text { High quality } \\
\text { streaming } \\
\text { audio }\end{array}$ & $\begin{array}{l}\text { Primarily } \\
\text { one-way }\end{array}$ & $\begin{array}{l}16-128 \\
\mathrm{kbit} / \mathrm{s} \\
\text { (Note } \\
3 \text { ) }\end{array}$ & $<10 \mathrm{~s}$ & $<<1 \mathrm{~ms}$ & $<1 \%$ PLR & \\
\hline Video & Videophone & Two-way & $\begin{array}{l}16-384 \\
\mathrm{kbit} / \mathrm{s}\end{array}$ & $\begin{array}{l}<150 \quad \mathrm{~ms} \\
\text { preferred } \\
\text { (Note } 4) \\
<400 \mathrm{~ms} \text { limit }\end{array}$ & & $<1 \%$ PLR & $\begin{array}{l}\text { Lip- } \\
\text { synch: } \\
<80 \mathrm{~ms}\end{array}$ \\
\hline Video & One-Way & $\begin{array}{l}\text { One- } \\
\text { Way }\end{array}$ & $\begin{array}{l}16-384 \\
\text { Kbit/s }\end{array}$ & $<10 \mathrm{~s}$ & & $<1 \%$ PLR & \\
\hline
\end{tabular}

NOTE 1: Assumes adequate echo control

NOTE 2 - Exact values depend on specific codec, but assumes use of a packet loss concealment algorithm to minimize effect of packet loss.

NOTE 3 - Quality is very dependent on codec type and bit-rate.

NOTE 4 - These values are to be considered as long-term target values which may not be met by current technology. 
Table 6 the ITU-T Performance targets for data applications [5], [6]

\begin{tabular}{|c|c|c|c|c|c|c|}
\hline Medium & Application & $\begin{array}{l}\text { Degree of } \\
\text { Symmetry }\end{array}$ & $\begin{array}{l}\text { Typical } \\
\text { Data }\end{array}$ & Key Perfo & $\begin{array}{r}\text { mance Paran } \\
\text { Values }\end{array}$ & ters \& Target \\
\hline & & & & $\begin{array}{c}\text { One-way } \\
\text { delay }\end{array}$ & $\begin{array}{c}\text { Delay } \\
\text { variation }\end{array}$ & $\begin{array}{c}\text { Information } \\
\text { loss }\end{array}$ \\
\hline Data & $\begin{array}{l}\text { Web-browsing - } \\
\text { HTML }\end{array}$ & $\begin{array}{l}\text { Primarily } \\
\text { one-way }\end{array}$ & $\sim 10 \mathrm{~KB}$ & $\begin{array}{l}\text { Preferred < } \\
2 \mathrm{~s} \\
\text { /page. } \\
\text { Acceptable } \\
<4 \mathrm{~s} \\
\text { /page }\end{array}$ & N.A. & Zero \\
\hline Data & $\begin{array}{l}\text { Bulk data } \\
\text { transfer/retrieval }\end{array}$ & $\begin{array}{l}\text { Primarily } \\
\text { one-way }\end{array}$ & $\begin{array}{l}10 \mathrm{~KB}- \\
10 \mathrm{MB}\end{array}$ & $\begin{array}{l}\text { Preferred < } \\
15 \mathrm{~s} \\
\text { Acceptable } \\
<60 \mathrm{~s}\end{array}$ & N.A. & Zero \\
\hline Data & $\begin{array}{l}\text { Transaction } \\
\text { services - high } \\
\text { priority e.g. } \\
\text { e-commerce, } \\
\text { ATM }\end{array}$ & Two-way & $<10 \mathrm{~KB}$ & $\begin{array}{l}\text { Preferred < } \\
2 \mathrm{~s} \\
\text { Acceptable } \\
<4 \mathrm{~s}\end{array}$ & N.A. & Zero \\
\hline Data & Command/control & Two-way & $\sim 1 \mathrm{~KB}$ & $<250 \mathrm{~ms}$ & N.A. & Zero \\
\hline Data & Still image & One-way & $\begin{array}{ll}< & 100 \\
\mathrm{~KB} & \end{array}$ & $\begin{array}{l}\text { Preferred < } \\
15 \mathrm{~s} \\
\text { Acceptable } \\
<60 \mathrm{~s}\end{array}$ & N.A. & Zero \\
\hline Data & Interactive games & Two-way & $<1 \mathrm{~KB}$ & $<200 \mathrm{~ms}$ & N.A. & Zero \\
\hline Data & Telnet & $\begin{array}{l}\text { Two-way } \\
\text { (asymmetric) }\end{array}$ & $<1 \mathrm{~KB}$ & $<200 \mathrm{~ms}$ & N.A. & Zero \\
\hline Data & $\begin{array}{l}\text { E-mail (server } \\
\text { access) }\end{array}$ & $\begin{array}{l}\text { Primarily } \\
\text { one-way }\end{array}$ & $<10 \mathrm{~KB}$ & $\begin{array}{l}\text { Preferred < } \\
2 \mathrm{~s} \\
\text { Acceptable } \\
<4 \mathrm{~s}\end{array}$ & N.A. & Zero \\
\hline Data & $\begin{array}{l}\text { E-mail server to } \\
\text { server transfer }\end{array}$ & $\begin{array}{l}\text { Primarily } \\
\text { one-way }\end{array}$ & $<10 \mathrm{~KB}$ & $\begin{array}{l}\text { several } \\
\text { Minutes }\end{array}$ & N.A. & Zero \\
\hline Data & Fax ("real-time") & $\begin{array}{l}\text { Primarily } \\
\text { one-way }\end{array}$ & $\sim 10 \mathrm{~KB}$ & $<30$ s/page & N.A. & $<10^{-6} \mathrm{BER}$ \\
\hline Data & $\begin{array}{l}\text { Fax (store \& } \\
\text { forward) }\end{array}$ & $\begin{array}{l}\text { Primarily } \\
\text { one-way }\end{array}$ & $\sim 10 \mathrm{~KB}$ & $\begin{array}{l}\text { several } \\
\text { Minutes }\end{array}$ & N.A. & $<10^{-6} \mathrm{BER}$ \\
\hline Data & $\begin{array}{l}\text { Low priority } \\
\text { Transactions }\end{array}$ & $\begin{array}{l}\text { Primarily } \\
\text { one-way }\end{array}$ & $<10 \mathrm{~KB}$ & $<30 \mathrm{~s}$ & N.A. & Zero \\
\hline Data & Usenet & $\begin{array}{l}\text { Primarily } \\
\text { one-way }\end{array}$ & $\begin{array}{l}>=1 \\
\mathrm{MB}\end{array}$ & $\begin{array}{l}\text { several } \\
\text { Minutes }\end{array}$ & N.A. & Zero \\
\hline
\end{tabular}

Based on the target performance requirements identified in table 5 and 6 , the various applications can be mapped onto axes of packet loss and oneway delay as shown in figure 2.1 . 


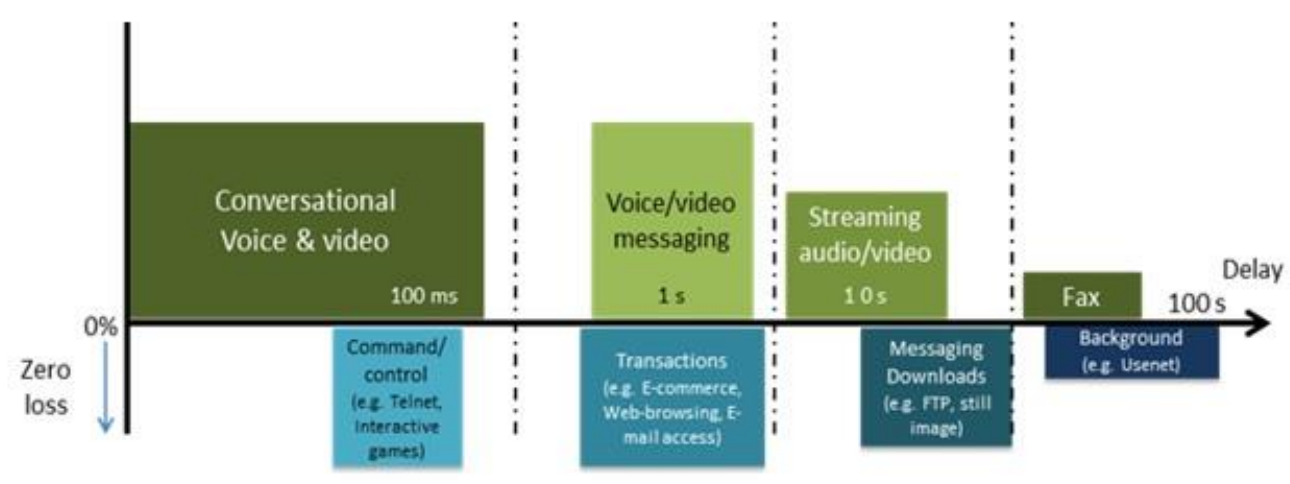

Fig 2.1. Target Values of ITU-T's QoS

\subsection{ETSI- QoS Parameters}

There is the need to specify independent QoS criteria for each service, thus the relevant prerequisites may be required.

Table 7 QoS Criteria of ETSI

\begin{tabular}{|l|l|l|}
\hline Service Independent QoS Criteria & Direct Services QoS Criteria & \multicolumn{1}{|c|}{$\begin{array}{l}\text { Store-and-Forward (S\&F) } \\
\text { Services QoS Criteria }\end{array}$} \\
\hline Radio network unavailability & File Transfer (FTP) & E-Mail \\
\hline Network non-accessibility & Mobile Broadcast & $\begin{array}{l}\text { Multimedia Messaging Service } \\
\text { (MMS) }\end{array}$ \\
\hline Attach failure ratio & Ping to talk over Cellular & Short Message Service (SMS) \\
\hline Attach Setup Time & $\begin{array}{l}\text { Push to } \\
\text { (PoC) }\end{array}$ & \\
\hline $\begin{array}{l}\text { PDP context activation failure } \\
\text { ratio }\end{array}$ & Streaming & \\
\hline PDP context activation time & Telephony & \\
\hline PDP Context Cut-off Ratio & Video Telephony & \\
\hline Data call access failure ratio & Web Browsing (HTT) & \\
\hline Data call access time & & \\
\hline
\end{tabular}

Table 8.1. Direct Services QoS Criteria -1 [2]

\begin{tabular}{|c|c|c|c|}
\hline $\begin{array}{l}\text { File Transfer } \\
\text { (FTP) }\end{array}$ & $\begin{array}{l}\text { Push to talk over Cellular } \\
\text { (PoC) }\end{array}$ & Video Telephony & Mobile Broadcast \\
\hline $\begin{array}{l}\text { Service Non- } \\
\text { Accessibility }\end{array}$ & Registration Failure Ratio & $\begin{array}{l}\text { Service } \\
\text { Accessibility }\end{array}$ & Network Non-Availability \\
\hline Setup Time & Registration Time & Service Access Time & $\begin{array}{l}\text { Service Discovery Failure } \\
\text { Ratio }\end{array}$ \\
\hline $\begin{array}{l}\text { IP-Service } \\
\text { Access Failure } \\
\text { Ratio }\end{array}$ & 3 Publish Failure Ratio & $\begin{array}{l}\text { Audio/Video } \\
\text { Failure Ratio }\end{array}$ & Service Discovery Time \\
\hline $\begin{array}{l}\text { IP-Service Setup } \\
\text { Time }\end{array}$ & Publish Time & $\begin{array}{l}\text { Audio/Video } \\
\text { Time }\end{array}$ & e Ratio \\
\hline $\begin{array}{l}\text { Session Failure } \\
\text { Ratio }\end{array}$ & $\begin{array}{l}\text { Registration } \\
\text { (long) }\end{array}$ & Cut-off Call Ratio & Bootstrapping Time \\
\hline Session Time & Registration Time (long) & $\begin{array}{l}\text { Speech Quality on Call } \\
\text { Basis }\end{array}$ & ESG Retrieval Failure Ratio \\
\hline Mean Data Rate & $\begin{array}{l}\text { Session Initiation Failure Ratio } \\
\text { (on-demand) }\end{array}$ & $\begin{array}{l}\text { Speech Quality on } \\
\text { Sample Basis }\end{array}$ & ESG Retrieval Time \\
\hline $\begin{array}{l}\text { Data Transfer } \\
\text { Cut-off Ratio }\end{array}$ & $\begin{array}{l}\text { Session Initiation Time (on- } \\
\text { demand) }\end{array}$ & Video Quality & Content Non-Accessibility \\
\hline
\end{tabular}


Table 8.2. Direct Services QoS Criteria -2 [2]

\begin{tabular}{|c|c|c|c|}
\hline Streaming & Telephony & Web Browsing (HTTP) & Ping \\
\hline $\begin{array}{ll}\text { Network } & \text { Non- } \\
\text { Availability } & \end{array}$ & Service Non-Accessibility & Service Non-Accessibility & Round Trip Time \\
\hline $\begin{array}{l}\text { Service Discovery } \\
\text { Failure Ratio }\end{array}$ & Setup Time & Setup Time & \\
\hline $\begin{array}{l}\text { Service } \\
\text { Time }\end{array}$ & Speech Quality on Call Basis & $\begin{array}{l}\text { IP-Service Access Failure } \\
\text { Ratio }\end{array}$ & \\
\hline $\begin{array}{l}\text { Bootstrapping Failure } \\
\text { Ratio }\end{array}$ & $\begin{array}{l}\text { Speech Quality on Sample } \\
\text { Basis }\end{array}$ & IP-Service Setup Time & \\
\hline Bootstrapping Time & Cut-off Call Ratio & Session Failure Ratio & \\
\hline $\begin{array}{l}\text { ESG Retrieval Failure } \\
\text { Ratio \& time }\end{array}$ & & Session Time & \\
\hline $\begin{array}{l}\text { Content } \\
\text { Accessibility }\end{array}$ & & Data Transfer Cut-off Ratio & \\
\hline \multicolumn{4}{|l|}{ Content Access Time } \\
\hline $\begin{array}{l}\text { Interactivity Response } \\
\text { Failure Ratio \& } \\
\text { Response time }\end{array}$ & & & \\
\hline
\end{tabular}

Table 9. Store-and-Forward (S\&F) Services QoS Criteria [2]

\begin{tabular}{|l|l|l|}
\hline \multicolumn{1}{|c|}{ E-Mail } & \multicolumn{1}{|c|}{$\begin{array}{c}\text { Multimedia Messaging Service } \\
\text { (MMS) }\end{array}$} & \multicolumn{1}{|c|}{ Short Message Service (SMS) } \\
\hline Service Non-Accessibility & Send Failure Ratio & Service Non-Accessibility \\
\hline Setup Time & Retrieval Failure Ratio & Access Delay \\
\hline $\begin{array}{l}\text { IP-Service Access Failure } \\
\text { Ratio }\end{array}$ & Send Time & End-to-End Delivery Time \\
\hline IP-Service Setup Time & Retrieval Time & Completion Failure Ratio \\
\hline Session Failure Ratio & Notification Failure Ratio & \\
\hline Session Time & Notification Time & \\
\hline Mean Data Rate & End-to-End Failure Ratio & \\
\hline Data Transfer Cut-off & End-to-End Delivery Time & \\
\hline Ratio & & \\
\hline
\end{tabular}

Table 10.1. ETSI QoS Parameters for VoIP -1

\begin{tabular}{|c|c|c|c|}
\hline $\begin{array}{l}\text { Qos Parameters } \\
\text { Specific To PLMN }\end{array}$ & $\begin{array}{l}\text { Call Delivery And } \\
\text { Collection }\end{array}$ & Internet Access & Other Services \\
\hline $\begin{array}{ll}\text { Unsuccessful } & \text { call } \\
\text { ratio } & \text { for } \\
\text { telephony } & \end{array}$ & $\begin{array}{l}\text { Unsuccessful call ratio } \\
\text { for call delivery }\end{array}$ & Login time & $\begin{array}{l}\text { Response times for } \\
\text { answering fault } \\
\text { reporting line }\end{array}$ \\
\hline \multirow[t]{4}{*}{ Dropped call ratio } & $\begin{array}{l}\text { Unsuccessful call ratio } \\
\text { with carrier selection }\end{array}$ & $\begin{array}{l}\text { Data transmission } \\
\text { speed achieved }\end{array}$ & Billing queries \\
\hline & $\begin{array}{l}\text { Blocking at Busy Hour } \\
\text { on the interconnection } \\
\text { circuit groups }\end{array}$ & $\begin{array}{l}\text { Unsuccessful data } \\
\text { transmission ratio }\end{array}$ & $\begin{array}{l}\text { Billing } \\
\text { amendments }\end{array}$ \\
\hline & & $\begin{array}{l}\text { Successful log-in } \\
\text { ratio }\end{array}$ & $\begin{array}{l}\text { Time to confirm } \\
\text { that a number can } \\
\text { or cannot be ported }\end{array}$ \\
\hline & & $\begin{array}{l}\text { Delay (one way } \\
\text { transmission time) }\end{array}$ & \\
\hline
\end{tabular}


Table 10.2. ETSI QoS Parameters for VoIP -2

\begin{tabular}{|l|l|}
\hline \multicolumn{1}{|c|}{$\begin{array}{c}\text { QoS parameters for voice, data and fax Mobile Services } \\
\text { accessed via the PSTN and SMS }\end{array}$} & \multicolumn{1}{|c|}{ Interconnection } \\
\hline Unsuccessful call ratio & $\begin{array}{l}\text { Supply time for initial physical } \\
\text { interconnection at a site }\end{array}$ \\
\hline Call set up time & $\begin{array}{l}\text { Supply time of E1 Interconnection circuits } \\
\text { on an established interconnection link }\end{array}$ \\
\hline Speech connection quality & $\begin{array}{l}\text { Supply time for a call by call carrier } \\
\text { selection facility to another operator }\end{array}$ \\
\hline Fax connection quality & $\begin{array}{l}\text { Supply time for handling customer } \\
\text { originated carrier pre-selection requests } \\
\text { from another operator }\end{array}$ \\
\hline Data rate of dial-up access to the Internet & $\begin{array}{l}\text { Availability of E1 interconnection circuits } \\
\text { per interconnection link }\end{array}$ \\
\hline Successful SMS Ratio & Fault rate per E1 interconnection circuit \\
\hline Completion Rate for SMS & $\begin{array}{l}\text { Fault repair time for the E1 interconnection } \\
\text { circuits }\end{array}$ \\
\hline Response time for admin/billing enquiries & \\
\hline Frequency of customer complaints & \\
\hline Customer complaints resolution time & \\
\hline Bill correctness complaints & \\
\hline Prepaid account credit correctness complaints & \\
\hline Bill presentation quality & \\
\hline Customer relations & \\
\hline Professionalism of help line & \\
\hline
\end{tabular}

\subsection{GPP Standard TS 23.207 Parameters}

Table 11.1. The3GPP QoS Parameters - 1 [7]

\begin{tabular}{|l|l|l|}
\hline RAB establishment success rate pS & Call drop rate & $\begin{array}{l}\text { Outgoing Hard Handover success } \\
\text { rate }\end{array}$ \\
\hline $\begin{array}{l}\text { RRC Connection Establishment Success } \\
\text { Rate }\end{array}$ & $\begin{array}{l}\text { Call Drop Rate } \\
\text { (Cell Level) }\end{array}$ & $\begin{array}{l}\text { Handover Success Rate (BSC and } \\
\text { Cell) }\end{array}$ \\
\hline UTRAN Service Access Success Rate & $\begin{array}{l}\text { SDCCH Blocking } \\
\text { Rate }\end{array}$ & \\
\hline $\begin{array}{l}\text { GERAN Service Access Success Rate for CS } \\
\text { Domain }\end{array}$ & TCH Blocking Rate & \\
\hline $\begin{array}{l}\text { GSM PDP Context Activation Success Rate } \\
\text { UMTS PDP Context Activation Success } \\
\text { Rate }\end{array}$ & & \\
\hline
\end{tabular}


Table 11.2. The3GPP QoS Parameters - 2 [9]

\begin{tabular}{|l|l|l|l|}
\hline \multicolumn{1}{|c|}{ Utilization KPI } & Availability KPI & \multicolumn{1}{|c|}{ Integrity KPI } \\
\hline $\begin{array}{l}\text { Percentage of Established } \\
\text { RABs, CS Speech }\end{array}$ & $\begin{array}{l}\text { UTRAN Cell } \\
\text { Availability }\end{array}$ & $\begin{array}{l}\text { Mean User Data Throughput } \\
\text { uplink on Iub interface (CS) }\end{array}$ & $\begin{array}{l}\text { Uplink Block Error } \\
\text { Rate }\end{array}$ \\
\hline $\begin{array}{l}\text { Percentage of Established } \\
\text { RABs, CS 64kbps } \\
\text { (Video telephony) }\end{array}$ & $\begin{array}{l}\text { Cell } \\
\text { Unavailability }\end{array}$ & $\begin{array}{l}\text { Mean User Data Throughput } \\
\text { downlink on Iub interface (CS) }\end{array}$ & TCH Traffic Carried \\
\hline $\begin{array}{l}\text { Percentage of Established } \\
\text { RABs, TotalPS }\end{array}$ & & $\begin{array}{l}\text { Mean User Data Throughput } \\
\text { uplink on Iub interface (PS) }\end{array}$ & \\
\hline & $\begin{array}{l}\text { Mean User Data Throughput } \\
\text { downlink on Iub interface (PS) }\end{array}$ & \\
\hline & $\begin{array}{l}\text { Mean User Data Throughput } \\
\text { uplink on IuCS interface }\end{array}$ & \\
\hline & $\begin{array}{l}\text { Mean User Data Throughput } \\
\text { downlink on IuCS interface }\end{array}$ & \\
\hline & $\begin{array}{l}\text { Mean User Data Throughput } \\
\text { uplink on IuPS interface }\end{array}$ & \\
\hline & $\begin{array}{l}\text { Mean User Data Throughput } \\
\text { downlink on IuPS interface }\end{array}$ & \\
\hline
\end{tabular}

\subsection{GSM Association Parameters}

Table 12.1 QoS Parameters of GSMA - 1 [6]

\begin{tabular}{|c|c|c|}
\hline Service Independent & $\begin{array}{l}\text { Short Message } \\
\text { Service }\end{array}$ & $\begin{array}{l}\text { Packet Switched Data Service (General } \\
\text { Packet Radio Service) }\end{array}$ \\
\hline $\begin{array}{l}\text { Network Accessibility } \\
\text { Circuit Switched }\end{array}$ & $\begin{array}{l}\text { Service Accessibility } \\
\text { SMS MO }\end{array}$ & $\begin{array}{l}\text { Service Accessibility Rate - Packet } \\
\text { Switched Data }\end{array}$ \\
\hline \multirow{3}{*}{$\begin{array}{l}\text { Network Accessibility } \\
\text { Packet Switched }\end{array}$} & Access Delay SMS MO & Set-up Time - Packet Switched Data \\
\hline & $\begin{array}{l}\text { End-to-End Delivery } \\
\text { Time SMS }\end{array}$ & Data Quality (DQ-PSD) \\
\hline & $\begin{array}{l}\text { Completion Rate SMS } \\
\text { Circuit switched }\end{array}$ & Completed Session Ratio \\
\hline
\end{tabular}

Table 12.2 QoS Parameters of GSMA - 2 [6]

\begin{tabular}{|c|c|c|}
\hline Telephony & $\begin{array}{l}\text { Circuit Switched Data } \\
\text { Service }\end{array}$ & $\begin{array}{c}\text { Data Service Class Definitions } \\
\text { and Measurements }\end{array}$ \\
\hline $\begin{array}{l}\text { Service Accessibility Telephony } \\
\text { (SA-T) }\end{array}$ & $\begin{array}{l}\text { Service Accessibility, Circuit } \\
\text { Switched Data }\end{array}$ & Conversational Class Data \\
\hline Setup Time Telephony (ST-T) & Set-up Time & Streaming Class \\
\hline CSSR (Call Setup Success Ratio) & Data Quality (DQ-CSD) & Interactive Class \\
\hline $\begin{array}{l}\text { REL (ISUPv2 } \\
\text { transparency) }\end{array}$ & $\begin{array}{l}\text { Completion Rate Circuit } \\
\text { Switched Data }\end{array}$ & Background class \\
\hline $\begin{array}{l}\text { OCN \& RDN (ISUPv2 Signaling } \\
\text { transparency) }\end{array}$ & & \\
\hline $\begin{array}{l}\text { Call Completion Rate Circuit } \\
\text { Switched telephony (CCR-CS-T) }\end{array}$ & & \\
\hline ALOC (Average Length of a Call) & & \\
\hline CLI Transparency & & \\
\hline Speech Quality (SpQ) & & \\
\hline
\end{tabular}


Author: Sigit Haryadi is lecturer in ITB (Bandung Institute of Technology), Indonesia, since 1984. Currently teaches several courses: Traffic Engineering, Quality and Regulatory Services Telecommunications and Regulations. In addition to teaching at the ITB, Sigit Haryadi also becomes experts at the Directorate General of Post and Telecommunication Indonesia since 1986. Experience in the industry: since 1990 to become an expert in some Providers and Telecom Operators in Indonesia. Since 2001, Sigit Haryadi became associate professor in the institution.

\section{References}

[1] Sigit Haryadi. (2013). Telecommunication Traffic: Technical and Business Consideration. Lantip Safari Media. Bandung, Indonesia.

[2] Sigit Haryadi. (2013). Telecommunication Service and Experience Quality. Lantip Safari Media. Bandung, Indonesia.

[3] ETSI Standard TS 102 250-2 v2-2-1. (2011). Speech and multimedia Transmission Quality (STQ); QoS aspects for popular services in mobile networks; Part 2: Definition of Quality of Service parameters and their computation.

[4] ITU-T Recommendation G-1010. (2011). End-user multimedia QoS categories. 2011.

[5] GSM Association Standard PRD IR 42. (2001). Definition of quality of service parameters and their computation. 2001.

[6] ITU-T Recommendation E.800. (1984). Definitions of terms related to quality of service.

[7] ITU-T Recommendation G.1000 (1984). Communications Quality of Service: A framework and definitions.

[8] 3GPP Standard TS 23-207 V5-10-0. (2005). Technical Specification. 3rd Generation Partnership Project.

[9] ETSI Standard EG 202-057 V1-3-1. (2008). Speech Processing, Transmission and Quality Aspects (STQ).

[10] 3GPP Standard TS 32-410. (2012). Telecommunication Management; Key Performance Indicators (KPI) for UMTS and GSM.

[11] 3GPP Standard TS 32.402. (2001). Telecommunication management; Performance Management (PM); Performance measurements. 2001. 
[12] European Telecommunications Standards Institute, ETSI TS 102 250-1 V2-2-1. (2011-04). Speech and multimedia Transmission Quality (STQ), QoS aspects for popular services in mobile networks; Part 1. Assessment of Quality of Service.

[13] Haryadi, Sigit; Limampauw, Ivantius. (2012). QoS Measurement of Telephony Services In 3G Networks Using Aggregation Method. Conference Proceeding of TSSA 2012. Denpasar, Indonesia.

[14] Haryadi, Sigit; Nusantara, Sandy. (2012). QoS Measurement of Web Browsing Services In $3 G$ Networks Using Aggregation Method. Conference Proceeding of TSSA 2012. Denpasar, Indonesia.

[15] Haryadi, Sigit; Pramudita, Arnold. (2012). QoS Measurement of Video Streaming Services in a 3G Networks Using Aggregation Method. Conference Proceeding of TSSA 2012. Denpasar, Indonesia.

[16] Haryadi, Sigit; Andina, Raisha. (2012). QoS Measurement of File Transfer Protocol Services In 3G Networks Using Aggregation Method. Denpasar, Indonesia. 2012.

[17] Hardy, C. William. (2001). QoS Measurement and Evaluation of Telecommunications Quality of Service. John Wiley \& Sons, Ltd. Baffins Lane, Chichester, West Sussex, PO19 1UD, England.

[18] ITU-T Recommendation G-107. (1984). The E-Model, a computational model for use in transmission planning.

[19] Haryadi, S. (2018, January 25). Chapter 1. The Concept of Telecommunication Network Performance and Quality of Service. Retrieved from osf.io/mukqb 